\title{
Laser-induced breakdown self-reversal isotopic spectrometry for isotopic analysis of lithium
}

\author{
Kévin Touchet ${ }^{1}$, Frédéric Chartier ${ }^{2}$, Jörg Hermann ${ }^{3}$, Jean-Baptiste Sirven ${ }^{1}$ \\ ${ }^{1}$ Den -Service d'Etudes Analytiques et de Réactivité des Surfaces (SEARS), CEA, Université Paris- \\ Saclay, 91191 Gif-sur-Yvette cedex, France \\ ${ }^{2}$ Den - Département de physico-chimie (DPC), CEA, Université Paris-Saclay, 91191 Gif-sur-Yvette \\ cedex, France \\ ${ }^{3}$ Aix-Marseille Université, CNRS, LP3, 13009 Marseille, France
}

\begin{abstract}
Online monitoring or in-situ isotopic analysis techniques in extreme environments are strategic tools in nuclear industry. A new optical method for performing isotopic analysis in solid samples at ambient pressure has been developed: Laser-Induced Breakdown self-Reversal Isotopic Spectrometry (LIBRIS). This method uses self-absorption of atomic or ionic resonance lines that are emitted from a non-uniform laser-induced plasma. It takes advantage of the fact that the spectral width of the absorption dip is much smaller than the spectral width of the emission line profile. Isotopic measurements were carried out on lithium samples by measuring the spectral position of the absorption dip that is shown to have a linear dependence on the ${ }^{6} \mathrm{Li}$ isotopic abundance. Stand-off and real-time analysis can be performed without any sample preparation or pre-treatment. The effect of the laser wavelength, of the ambient gas and of the gate delay is investigated. Optimum conditions lead to a relative uncertainty of about $6 \%$ on the isotopic abundance measurement of ${ }^{6} \mathrm{Li}$. The influence of the spectral shifts due to Stark and Doppler effects on the performance of LIBRIS are discussed.
\end{abstract}

Keywords: isotopic analysis; absorption spectrometry; lithium; LIBS; LIBRIS; self-absorption

\section{Introduction}

Isotopic analysis is essential in different domains and in particular in the nuclear energy field $[1,2]$ and for non-proliferation of nuclear materials [3, 4]. Reference techniques include Thermo-Ionization Mass Spectrometry (TIMS) and Multi-Collection Inductively Coupled Plasma Mass Spectrometry (MC-ICP/MS) [3, 5], enabling measurements with high trueness, precision and sensitivity [3, 5, 6]. Glow Discharge Mass Spectrometry (GD-MS) and Laser Ablation coupled with Inductively Coupled Plasma-Mass Spectrometry (LA-ICP-MS) are also used for elemental or isotopic analyses directly on solid samples with high performance [5].

The coupling of optical measurements with laser ablation techniques, like in Laser-Induced Breakdown Spectroscopy (LIBS), has ideal characteristics for stand-off in-situ or online monitoring [7] at atmospheric pressure [8, 9, 10] for elemental analyses.

Due to its capabilities, LIBS is used in numerous fields like geology for in-situ chemical determination of minerals and rocks $[11,12]$. In the nuclear energy field, it was developed for onsite control of nuclear components [13], for nuclear forensics purposes [8] [14], or for fusion applications [15]. 
Benefits of this laser-based technique include the rapid and direct elemental characterization of solid samples without the requirement of chemical dissolution procedures [7]. However, plasmas induced by laser ablation at atmospheric pressure are characterized by large values of electron number density causing significant Stark broadening of spectral lines. Thus, according to the small isotopic shift, the isotopic lines can hardly be distinguished. In this case, even if the spectral resolution is not enough to separate the lines, a spectral shift of the line maximum can sometimes be observed when the isotopic ratio changes [9].

Consequently, the analysis is sometimes realized in vacuum or under reduced pressure [16, 17]. In such conditions, the electron number density is reduced and the spectral broadening low enough to observe the individual isotopic lines. Nevertheless, these experimental conditions are incompatible with in-situ measurements.

Other techniques can be used for isotopic analysis based on laser ablation such as Laser Ablation-Absorption Spectroscopy (LAAS) [18] and Laser-Induced Breakdown Spectroscopy coupled with Laser-Excited Atomic Fluorescence (LIBS/LEAF) [19]. Nevertheless, these techniques perform better under vacuum. Moreover, both techniques need a tunable laser and the configuration of lasers adds additional complexity in the experimental setup. Finally, the use of a laser for measuring the absorption or induced fluorescence ensures good sensitivity, but the analysis is specific to a single chemical element.

A powerful alternative is the so-called Laser Ablation Molecular Isotopic Spectrometry (LAMIS), which measures the spectra from molecular species present when the plasma cools down. The molecular emission is typically characterized by larger isotopic shifts, compared to atomic or ionic emission. This technique was employed to measure boron isotopes in pressedpowder disks of boron nitride and boron oxide for example [20, 21]. The boron isotopic shift makes it possible to analyze boron with good precision [22]. However, the isotopic shift of the molecular form is not always significantly larger than the isotopic shift of the atomic or ionic form. For example, the isotopic shift of molecular emissions of uranium oxide is only slightly larger (35 pm around $593 \mathrm{~nm}$ ) than the isotopic shift of the ionic form of uranium (24.8 pm at $424.43 \mathrm{~nm}$ ) [10]. In addition, molecular emission is not always observable in the laser-induced plasma. For example, in the case of lithium, the molecular emission does not occur because of the low dissociation energies of the lithium-containing molecular species.

This paper presents a method called Laser Induced Breakdown self-Reversal Isotopic Spectrometry (LIBRIS) that exploits the fact that the spectral width of the absorption dip of selfreversed resonance lines is much smaller than the width of the emission line profile [23]. Like for emission peaks [9], the absorption dip wavelength shifts when the isotopic ratio varies, which was observed in [24] the case of the lithium $670.776 \mathrm{~nm}$ line. Therefore, isotopic analysis is possible from the precise determination of this wavelength. Based on the linear dependence of the spectral position of the absorption dip versus the ${ }^{6} \mathrm{Li}$ isotopic abundance, the feasibility is demonstrated and the analytical performances of LIBRIS are evaluated. The effect of the laser wavelength, of the ambient gas and of the gate delay is investigated. The influence of the spectral shifts due to Stark and Doppler effects on the LIBRIS performance are discussed.

\section{Materials and methods}

\subsection{Experimental setup}

Experiments were carried out with two different LIBS systems: one with a frequencyquadrupled Nd:YAG laser (Quantel, model Ultra) at a wavelength of $266 \mathrm{~nm}$ and the other with a fundamental-frequency Nd:YAG laser (Quantel, model Brio) at a wavelength of $1064 \mathrm{~nm}$. 
Both laser sources delivered pulses of $4 \mathrm{~ns}$ duration with an energy set to $5.3 \pm 0.3 \mathrm{~mJ}$. The laser beams were focused onto the sample surface using a plano-convex lens of $200 \mathrm{~mm}$ focal length. With a spot diameter of $60 \mu \mathrm{m}$ of the Gaussian beam, a laser fluence of about $190 \pm$ $11 \mathrm{~J} / \mathrm{cm}^{2}$ at $266 \mathrm{~nm}$ and $1064 \mathrm{~nm}$ was obtained on the sample surface.

Spectroscopic measurements were performed with a spectrometer of $1 \mathrm{~m}$ focal length (Jobin Yvon, model THR1000) equipped with a diffraction grating of 2400 lines $/ \mathrm{mm}$ and coupled to an intensified charge-coupled device (ICCD) matrix detector (Andor, model iStar) for timeresolved measurements. The instrumental spectral width at the wavelength of $670.776 \mathrm{~nm}$ was measured to $12.5 \pm 0.5 \mathrm{pm}$ (full width at half-maximum) using a Hollow Cathode Lamp. The plasma emission was captured collinearly with the laser using an achromatic telescope with a magnification factor of $1 / 4$ and an optical fiber of $910 \mu \mathrm{m}$ core diameter that was coupled to the spectrometer.

\subsection{Samples}

The samples were prepared by mixing two different powders of lithium carbonate (Sigma Aldrich; CAS number: 554-13-2). The first had natural isotopic abundance (7.5 \pm 0.2 at. \% of ${ }^{6} \mathrm{Li}$ ) and the other was highly enriched in ${ }^{6} \mathrm{Li}$ (95.49 \pm 0.05 at. \%). The lithium isotopic abundance of the powder mixtures used for the various samples was measured by ICP-MS/MC (ThermoFisher Scientific, model Neptune). A pelletizer (ICL Die sets with $13 \mathrm{~mm}$ diameter) was used to realize pellets of lithium carbonate with the required isotopic ratio. The powder mixture was pressed with a pressure force of $10 \mathrm{kN}$ during 10 minutes.

These pellets were placed on a motorized sample holder in a chamber. During the experiments, the chamber was filled with air or argon at atmospheric pressure.

\subsection{Measurement and treatment methods}

Ten laser shots were performed for each crater which led to one spectrum. On each sample, 100 craters were realized for a total of 100 spectra, averaged 25 by 25 , which allowed to obtain 4 repetitions per sample.

The precise wavelength of the self-reversed line was ascertained by fitting the observed line shape. The shape of the lithium spectral line is a convolution between Gaussian and Lorentz profiles (Voigt function). Various broadening phenomena exist: instrumental, Doppler effects for the Gaussian contributions [25] and natural, resonant, pressure broadenings and Stark effect for the Lorentzian contributions. In our case, the line could be approximated by a Lorentz profile because of an important Stark broadening [26, 27, 28] compared to the Gaussian contributions. Then, the fitting curve was composed of a background term (continuous value) and two Lorentzian functions (one positive and a second negative: emission and absorption dip respectively). The estimated accuracy on the spectral position of the absorption dip was estimated to about $0.4 \mathrm{pm}$ ( $95 \%$ of confidence and 30 repetitions). According to the estimated accuracy, the minimum detectable shift is therefore estimated at $0.6 \mathrm{pm}(3 \sigma, 99.7 \%$ of confidence).

\section{Results and discussion}

\subsection{LIBRIS principle}

In the case of a non-uniform plasma (especially in ambient air [29]), the plasma can be schematized in two distinct zones: the hot plasma core with high electron density and the cold border with lower electron density (See Fig. 1 (A) and (B)). 


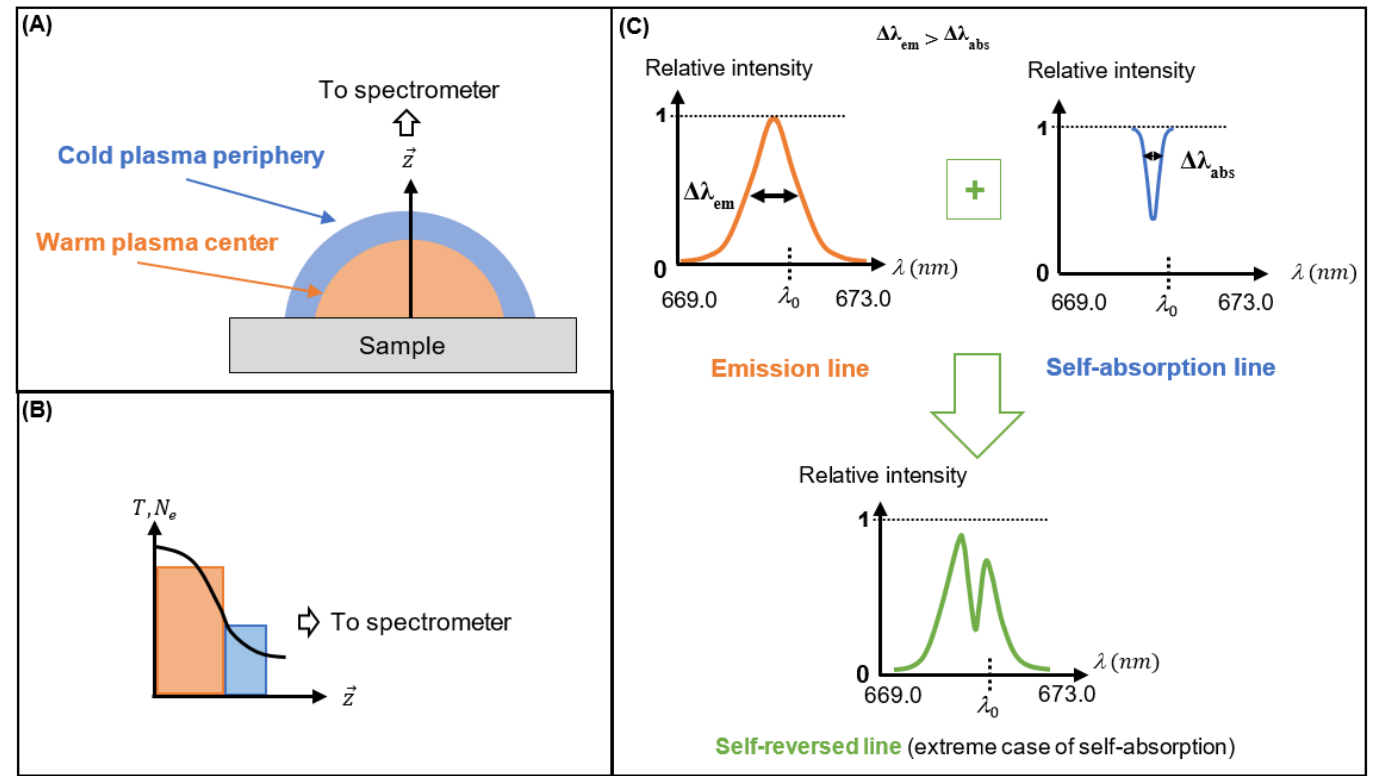

Figure 1: (A) Schematic of a non-uniform plasma comprising a hot core and a cold periphery. (B) Schematic graph of the physical characteristics (electron number density $N_{e}$ and electron temperature $T$ ) as functions of the distance from the sample surface $(\vec{z})$. (C) The optical phenomena in the plasma: emission from the plasma core, absorption from the border, and the line profile resulting from both effects. The resonance wavelength of the transition is labelled $\lambda_{0}$. The asymmetry is attributed to Stark shifting towards the blue.

During a LIBS measurement, two phenomena appear from these separate spatial zones [30]. The hot plasma core volume generates an intense and strongly Stark-broadened line emission. The core emission is partially absorbed by the cold plasma border. The absorption is particularly strong for resonance lines due to the large electron number density of the ground state atoms in the cold border. The absorption profile has a lower spectral width due to reduced Stark broadening in the plasma border of lower electron density.

As the result of these two phenomena, the observed line profile, exhibits an absorption-dip if the self-absorption in the plasma border is strong enough (See Fig. 1 (B)), and from the line fitting the absorption dip wavelength can be more accurately determined than the emission one.

The LIBRIS method exploits this self-absorption phenomenon by measuring the spectral position of the absorption dip that increase from the wavelength of the ${ }^{7} \mathrm{Li}$ transition toward the ${ }^{6} \mathrm{Li}$ transition when the ${ }^{6} \mathrm{Li}$ abundance varies from 0 to $100 \%$. This is illustrated in Fig. 2 (A) where the absorption dip is shown for three samples having different isotopic abundances. The spectral position of the absorption dip $\lambda_{a b s}$ is displayed as a function of the ${ }^{6} \mathrm{Li}$ abundance in Fig. 2 (B). A significant shift can be observed when the ${ }^{6} \mathrm{Li}$ abundance increases from natural abundance (7.5 at. \%) to 95.53 at. \%, and a linear dependence is observed between the wavelength shift and the ${ }^{6} \mathrm{Li}$ isotopic abundance. 


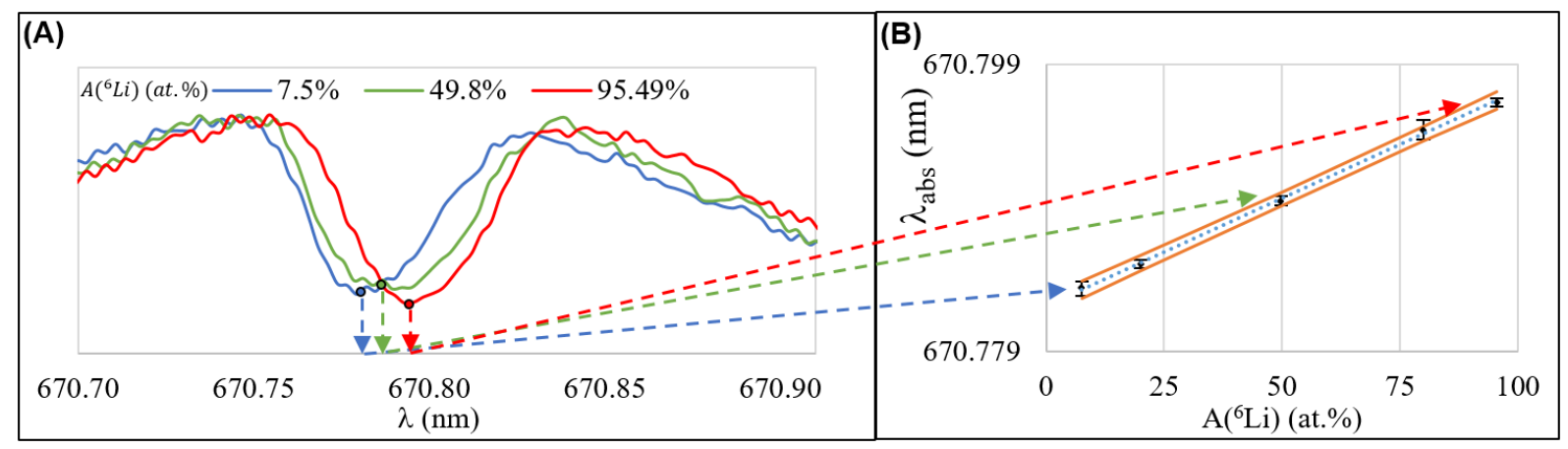

Figure 2: LIBRIS principle. (A) LIBRIS spectra with three isotopic abundances of lithium 6. (B) Lithium calibration curve in the LIBRIS method.

The LIBRIS method is thus based on this linear correlation with the self-reversed peak for the isotopic characterization as shown in Fig. 2. The central wavelength of the absorption dip is given by

$$
\lambda_{a b s}=a * A\left({ }^{6} L i\right)(a t . \%)+b
$$

Equation 1

where $A\left({ }^{6} \mathrm{Li}\right)($ at. \%) is the isotopic abundance of lithium 6 in atomic percentage.

The slope $a$ is the isotopic shift per unit abundance of the ${ }^{6} \mathrm{Li}$ isotope (in pm/\%), for which the theoretical value is $a_{T h}=\frac{I S(\mathrm{pm})}{100}$ where IS is the isotopic shift between ${ }^{6} \mathrm{Li}$ and ${ }^{7} \mathrm{Li}$. The intercept $b$ is the wavelength of the ${ }^{7} \mathrm{Li}$ line that would be observed for $a{ }^{6} \mathrm{Li}$-free sample (in nm).

From this principle, it is clear that the method enables the isotopic analysis of elements where only two isotopes are present in the sample, such as lithium, or if other isotopes are present in negligible proportions.

The most appropriate lithium transition is the resonance line $\mathrm{Li}$ I $2 s^{2} \mathrm{~S}-2 p^{2} \mathrm{P}^{\circ}$ at $670.7 \mathrm{~nm}$ that has the largest isotopic shift of $15.8 \pm 0.3 \mathrm{pm}$ [31, 32]. This line is a resonant doublet at $670.7760 / 670.7911 \mathrm{~nm}$ for ${ }^{7} \mathrm{Li}$ (difference of $15.1 \mathrm{pm}$ ), $670.7918 / 670.8068 \mathrm{~nm}$ for ${ }^{6} \mathrm{Li}$ (difference of $15.0 \mathrm{pm}$ ) [33]. For both isotopes the blue line of the doublet is twice as intense as the red one [34].

\subsection{Optimization of experimental parameters}

Many parameters affect the LIBS signal [35]. In this study, the selected preponderant parameters are (i) the laser wavelength, (ii) the background gas atmosphere and, (iii) the delay and the width of the detector gate. Then, to find the experimental conditions for optimized LIBRIS measurements, we proceeded in two steps. The first step consisted in minimizing the FWHM of the self-reversed peak in order to measure its central wavelength as accurately as possible. The second step aimed at characterizing the expanded relative uncertainty (at $95 \%$ of confidence and for 4 repetitive measurements) of quantification of the sample with ${ }^{6} \mathrm{Li}$ isotopic abundance of 50 at. \%, noted $U_{50}$. 


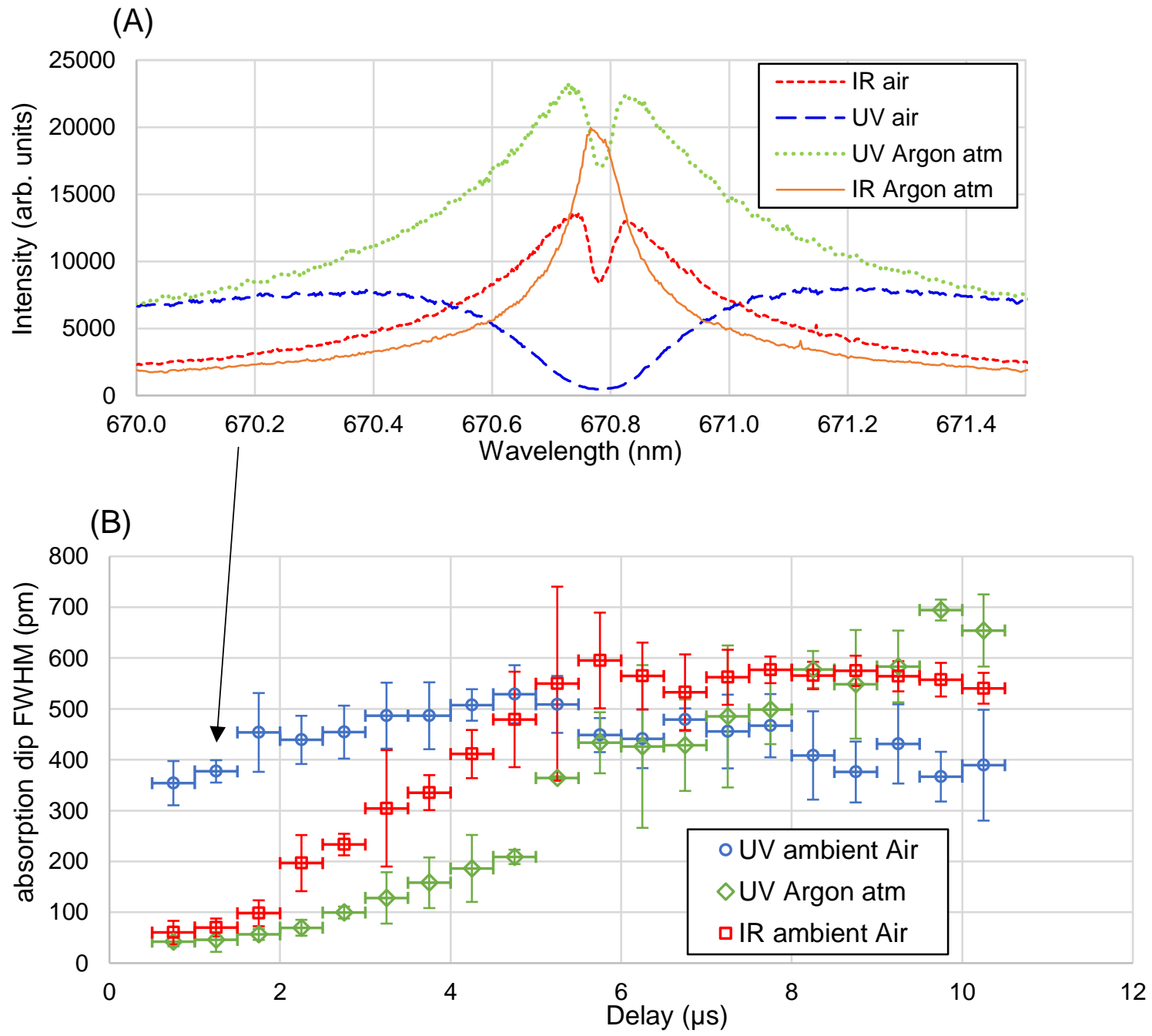

Figure 3: (A) Spectrum of a Li (I) line with different experimental conditions (each spectrum is the average of 1000 shots), a temporal window of $1 \mu$ s of delay and $500 \mathrm{~ns}$ of gate width. (B) Temporal evolution of the Full Width at Half Maximum (FWHM) of the absorption dip for the different experimental conditions. Each value was obtained by averaging over 5 spectra recordings, each spectrum being the result of 30 laser pulses. The horizontal error bars correspond to the gate width of $500 \mathrm{~ns}$. The vertical error bars correspond to a $95 \%$ confidence interval estimated from the 5 measurement replicas.

Each experimental condition leads to a different spectral profile of the atomic lithium line (see Fig. $3(\mathrm{~A})$ ). The emission and absorption FHWM give qualitative information about the electron number density in the emission and absorption layers of the plasma.

The temporal evolution of the FWHM of the absorption dip is shown in Fig.3 (B) as a function of the delay for different experimental conditions. In the case of ablation with the IR laser in argon atmosphere the lithium line is no longer self-reversed, as shown in Fig. $3(\mathrm{~A})$. Thus, the corresponding curve is not presented in Fig. $3(\mathrm{~B})$ and this experimental condition will not be considered further.

\subsubsection{Influence of the laser ablation wavelength}

At a delay of $1 \mu$ s (see Fig.3 (A)), the emission lines observed for UV laser ablation in ambient air and in argon had significant FWHM (2100 4400 pm and $430 \pm 50$ pm, respectively). In the case of IR laser ablation in ambient air and in argon the spectral widths of the emission line 
were lower (respectively $240 \pm 25 \mathrm{pm}$ and $\approx 180 \mathrm{pm}$ ). These observations were coherent with the literature: the higher spectral width of the emission line with the UV laser ablation is explained by a lower absorption of the laser pulse by the plasma, resulting in a better ablation efficiency and a higher electron number density [30, 36].

The absorption dip observed for UV laser ablation in ambient air has a large FWHM $(375 \pm 25$ pm) compared to that observed for UV laser ablation in argon atmosphere (63 $\pm 10 \mathrm{pm}$ ) and for IR laser ablation in ambient air $(60 \pm 9 \mathrm{pm})$ (see Table 1). This means that the electron number density is larger in the plasma periphery in the first case than in the other cases.

\subsubsection{Influence of the ambient gas}

The nature of the ambient gas has a huge influence on the characteristics and uniformity of the plasma. The argon atmosphere and ambient air are interesting because both have a strongly different effect on the laser-produced plasma [30], see Fig. 1. In argon atmosphere, the plasma is spatially more uniform in density and temperature (see Fig. $3(\mathrm{~A})$ ). This can be attributed to the formation of an isolating layer at the vapor-gas contact front [37]. Argon atoms have a large energy gap between the ground state and the first excited state $(11.55 \mathrm{eV})$, wherefore the rates of collisional excitation and deexcitation are very small and the plasma electrons interact with argon atoms mostly through elastic collisions thereby conserving their energy.

On the other hand, ambient air is composed of molecules and for this reason inelastic collisions prevail due to the numerous rovibrational molecular levels. The ablation plume is cooled down at the border and a non-uniform plasma is obtained. Moreover, compared to ambient air, the plasma in argon has a cold border of smaller thickness because, in the latter, the plasma is more uniform and therefore the cold zone is a thin layer of ionized gas heated by the shock wave, causing the path length in the absorbent zone to be shorter. From the fit of the line profile, the amplitude ratio between the emission and self-reversed peaks $\frac{I_{\text {self-reversed }}}{I_{\text {emission }}}$ enables to define a transmission coefficient $T(\%)=\left(1-\frac{I_{\text {self-reversed }}}{I_{\text {emission }}}\right) * 100$. This coefficient is lower in the case of UV laser ablation in argon atmosphere as opposed to UV laser in ambient air (34 $\%$ and $95 \%$, respectively, at a gate delay of $1 \mu \mathrm{s})$.

For IR laser ablation, this phenomenon and the lower density in the plasma lead to the same observations but there are more pronounced in this case. Indeed, with the IR laser ablation in ambient air, there is a self-reversed line with a transmission coefficient of $46 \%$. In argon atmosphere no self-reversed line is observable whatever the gate delay (Fig. $3(A)$ ).

\subsubsection{Influence of the temporal window}

The most appropriate delay seemed to be at the lowest values (500 ns or $1 \mu \mathrm{s})$ in the case of UV ablation in argon atmosphere and of IR ablation in ambient air (see Fig. 3 (B)), because the peak widths were almost 10 times smaller than the longest delay.

In the case of UV ablation in ambient air, a slight variation of the FWHM was observed. The lowest values were found for the shortest (FWHM=354 $\pm 35 \mathrm{pm}$ at $500 \mathrm{~ns}$ ) or longest delay $(\mathrm{FWHM}=389 \pm 88 \mathrm{pm}$ at $10 \mu \mathrm{s})$. However, at short delay the signal was obviously much higher than at long delay due to a higher plasma temperature.

\subsection{Evaluation of analytical performances}

The different analyses of lithium were carried out at short delay $(1 \mu \mathrm{s})$ and with a gate width of half-delay (500 ns) in the three different experimental conditions described. As the gate width 
is an important parameter, we selected a gate width of half-delay because it is a good compromise between enough signal and weak variations of physical characteristics of the plasma during the measurement. Using a higher gate width leads to a distorted spectral profile. Then in the worst case, the Stark and Doppler shifts variations during the measurement induces a flattened absorption dip and decrease the wavelength determination accuracy. A higher gate width than the half delay also leads to a convolution of the spectral width because of significant variations of physical characteristics of the plasma, like the electron number density, during the measurement.

Three calibration curves were obtained: with an ultraviolet laser (266 nm) in ambient air and in argon atmosphere, and with an infrared laser (1064 nm) in ambient air. Table 1 gathers different figures of merit of calibration curves obtained.
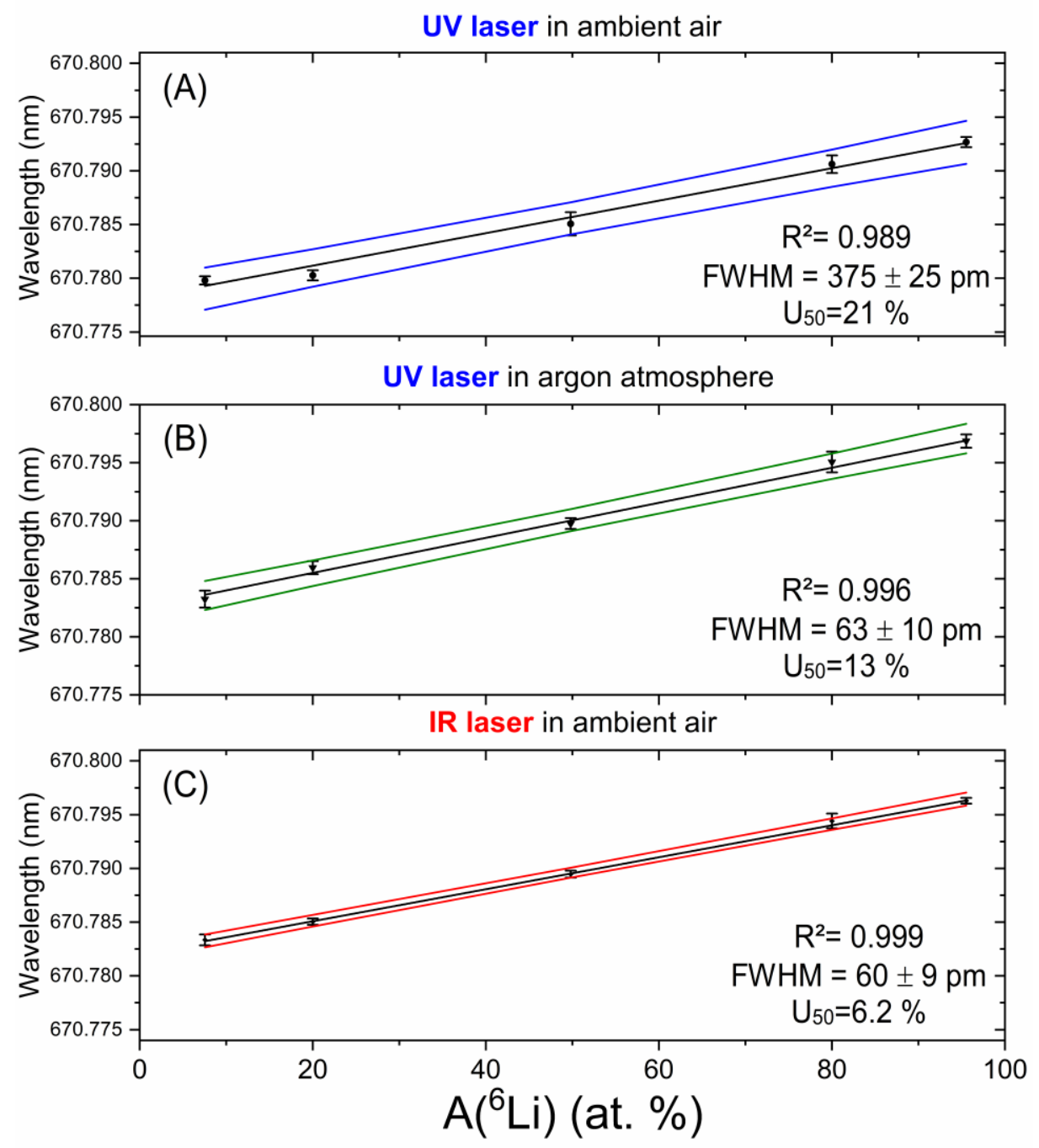

Figure 4: LIBRIS calibration curves and confidence hyperboles (95\% of confidence and 4 repetitions). (A) UV laser ablation in ambient air. (B) UV laser ablation in argon atmosphere. (C) IR laser ablation in ambient air. Wavelength of the maximum of the self-reversed peak as a function of the isotopic abundance $A\left({ }^{6} L i\right)(a t . \%)$.

In the case of LIBRIS analysis with IR laser ablation in ambient air (see Fig. $4(\mathrm{C})$ ), the FWHM of the self-reversed peak was low $(60 \pm 9 \mathrm{pm})$ and the correlation coefficient close to $1\left(\mathrm{R}^{2}=\right.$ 
0.999). The relative uncertainty at 50 at. \% of ${ }^{6} \mathrm{Li}\left(\mathrm{U}_{50}\right)$ reached $6.2 \%$. In the two other conditions, this uncertainty was higher.

In Table 1 we see that all experimental slopes corresponded to the expected value $\left(a\left(\frac{p m}{\%}\right)_{T h}=\right.$ $\left.(15.8 \pm 0.3) * 10^{-2} \mathrm{pm} / \%\right)$, whereas the experimental intercepts were significantly different from the expected value $b(\mathrm{~nm})_{T h}=670.7810 \mathrm{~nm}$, corresponding to the center wavelength of the unresolved ${ }^{7} \mathrm{Li}$ doublet lines.

In the case of UV laser in ambient air, there occurred a blue shift of $-3 \mathrm{pm}$. For UV laser in argon atmosphere and IR laser in ambient air, we observed red shifts of $+1.4 \mathrm{pm}$ and $+1.1 \mathrm{pm}$, respectively.

The spectral shift can be explained by the Stark and the Doppler effects. The Li I $670.7 \mathrm{~nm}$ transition is Stark-shifted towards the blue by about $-4 \mathrm{pm}$ for $N_{e}=1 \times 10^{17} \mathrm{~cm}^{-3}$ [38]. The electron density in the cold plasma border being typically lower than $1 \times 10^{17} \mathrm{~cm}^{-3}$ (see simulation of absorption dip reported in Ref. 30 ) the observed maximum blue shift of $-3 \mathrm{pm}$ can be attributed to the Stark effect. To explain the red shift observed in two cases, we assumed that during the temporal gate of measurement, the lithium atoms had an average speed in the plasma center slower than in the plasma periphery, see Fig. 1 (B) [39]. Consequently, the plasma periphery moved away from the plasma core. This relative movement induced a red shift of the absorption dip by the Doppler Effect. For a typical plume expansion velocity of the order of $10^{3} \mathrm{~m} \cdot \mathrm{s}^{-1}$, a red shift of about $2.1 \mathrm{pm}$ for the absorption dip was expected.

In the case of the IR laser in ambient air, the experimental intercept was the closest to the expected value. This was due to the Stark (blue shift) and the Doppler (red shift) effects compensating one another.

Table 1: Analytical figures of merit under different experimental conditions: The correlation coefficient $\left(R^{2}\right)$, the relative uncertainty of quantification with $95 \%$ of confidence and 4 repetitions at 50 at. \% of ${ }^{6} \mathrm{Li}\left(U_{50}\right)$, the full width at half-maximum of the absorption dip (FWHM), the slope and intercept of the calibration curves a $(\mathrm{pm} / \%)^{*} 10^{-2}$ and $\mathrm{b}(\mathrm{nm})$ respectively.

\begin{tabular}{|c|c|c|c|c|c|}
\hline & $\mathrm{R}^{2}$ & $U_{50}(\%)$ & FWHM (pm) & $\mathrm{a}(\mathrm{pm} / \%)^{*} 10^{-2}$ & $\mathrm{~b}(\mathrm{~nm})$ \\
\hline UV air & 0.989 & 21 & $375 \pm 25$ & $15.5 \pm 3.0$ & $670.7779 \pm 0.0018$ \\
\hline UV argon atm & 0.996 & 13 & $63 \pm 10$ & $15.4 \pm 1.9$ & $670.7824 \pm 0.0011$ \\
\hline IR air & 0.999 & 6.2 & $60 \pm 9$ & $15.0 \pm 0.9$ & $670.7821 \pm 0.0005$ \\
\hline & & & $\begin{array}{c}\text { Expected } \\
\text { values }\end{array}$ & $15.8 \pm 0.3[31]$ & 670.7810 \\
\hline
\end{tabular}

Table 1 shows that the worst analytical performance $\left(U_{50}\right)$ is obtained when the absorption dip width is the highest, i.e. with the UV laser in ambient air. This is consistent with our initial approach consisting in minimizing the absorption width to optimize the analysis. However, in the two other cases the FWHM of the absorption dip are the same but $U_{50}$ is significantly different. This means that the effect of the Stark broadening, mainly responsible for the peak width, on the analysis, is not straightforward. Obviously, other factors have an influence, such as the plasma repeatability for instance.

On the contrary, the influence of the Stark or Doppler shift is very clear, since it induces a systematic error on the measured wavelength. As the total isotopic shift between ${ }^{6} \mathrm{Li}$ and ${ }^{7} \mathrm{Li}$ is $15.8 \mathrm{pm}$, a $1 \mathrm{pm}$ shift leads to an error of $6.3 \%$ on the isotopic abundance. This shift depends 
on the matrix and on measuring conditions. A major consequence for the LIBRIS technique is that standard samples are therefore needed for calibration.

It should be noted that in the case of IR laser ablation in argon atmosphere, for which the line is not self-reversed, the use of the emission peak wavelength allows the isotopic quantification of ${ }^{6} \mathrm{Li}$ [9]. Using a curve fitting with one positive Lorentz function and a background term, we obtained a relative uncertainty of quantification $\left(\mathrm{U}_{50}\right)$ of $12 \%$. It is an equivalent analytical performance to that of UV laser ablation under argon using absorption dip. This is possible thanks to a relatively low FWHM of the emission peak (around $180 \mathrm{pm}$ ). However, we also observed a significant blue shift of about $-3.6 \mathrm{pm}$ in this experimental case. This important shift is due to the Stark and the Doppler effects. As mentioned previously, the Stark effect induces a blue shift of the line. But contrary to the absorption dip, the emission peak undergoes a Doppler shift towards the blue because the emitting lithium atoms move towards the observer.

\section{Conclusion}

Laser-Induced Breakdown self-Reversal Isotopic Spectrometry (LIBRIS) is introduced for isotopic analysis of solid samples at ambient pressure. This method uses the linear correlation between the absorption dip wavelength of a self-reversed line and isotopic abundance. In this paper it was successfully applied to isotopic analysis of lithium.

A parametric study was carried out with the aim to improve the analytical performances. The influence of the laser wavelength, of the ambient gas and of the detection temporal parameters was investigated. The optimization was realized in two steps. The first step concerned the selection of the temporal window where the FWHM of the absorption dip was the lowest. In the second step, the analytical performance under the different experimental conditions were evaluated. Optimum conditions lead to a relative uncertainty of about $6 \%$ on the isotopic abundance of ${ }^{6} \mathrm{Li}$.

Spectral shifts induced by Stark and Doppler Effects were observed on the experimental intercepts of the calibration curves. It is important to note that these spectral shifts depend on plasma parameters, and change with experimental conditions and with the sample matrix. Therefore, calibration using matrix-matched samples is required for the isotopic abundance determination.

The presented approach can be extended to any other self-reversed line or emission peak with a low spectral width of a chemical element with a sufficiently large isotopic shift. In a future work, possibilities and limitations of the LIBRIS technique will be investigated for other elements of interest in the nuclear industry.

Moreover, the use of the emission peak wavelength for the isotopic quantification of ${ }^{6} \mathrm{Li}$ has the advantage of allowing the analysis with a lower lithium concentration [9] and thus expands the scope of potential applications of this technique.

\section{Acknowledgement}

We thank Hélène Isnard for helpful discussions and isotopic analysis of lithium samples by Multi-Collection Inductively Coupled Plasma Mass Spectrometry. 
[1] I. W. Croudace, B. C. Russell, P. W. Warwick, Plasma source mass spectrometry for radioactive waste characterisation in support of nuclear decommissioning: a review, J. Anal. At. Spectrom. 32 (2017) 494-526, doi: 10.1039/C6JA00334F.

[2] W. Bu, Y. Ni, G. Steinhauser, W. Zheng, J. Zheng, N. Furuta, The role of mass spectrometry in radioactive contamination assessment after the Fukushima nuclear accident, J. Anal. At. Spectrom. 33 (2018) 519-546, doi: 10.1039/C7JA00401J.

[3] F. E. Stanley, A. M. Stalcup, H. B. Spitz, A brief introduction to analytical methods in nuclear forensics, J. Radioanal. Nucl. Chem. 295 (2013) 1385-1393, doi: 10.1007/s10967-012-19273 .

[4] D. L. Donohue, Strengthening IAEA safeguards through environmental sampling and analysis, J. Alloys Compd. 271-273 (1998) 11-18, doi: 10.1016/S0925-8388(98)00015-2.

[5] N. Jakubowski, T. Prohaska, L. Rottmann, F. Vanhaecke, Inductively coupled plasma- and glow discharge plasma-sector field mass spectrometry Part I. Tutorial: Fundamentals and instrumentation, J. Anal. At. Spectrom. 26 (2011) 693-726, doi: 10.1039/C0JA00161A.

[6] J. Harvey, E. F. Baxter, An improved method for TIMS high precision neodymium isotope analysis of very small aliquots (1-10 ng), Chem. Geol. 258 (2009) 251-257, doi: 10.1016/j.chemgeo.2008.10.024.

[7] S. S. Harilal, B. E. Brumfield, N. L. LaHaye, K. C. Hartig, M. C. Phillips, Optical spectroscopy of laser-produced plasmas for standoff isotopic analysis, Appl. Phys. Rev. 5 (2018) 21301, doi: $10.1063 / 1.5016053$.

[8] F. R. Doucet, G. Lithgow, R. Kosierb, P. Bouchard, M. Sabsabi, Determination of isotope ratios using Laser-Induced Breakdown Spectroscopy in ambient air at atmospheric pressure for nuclear forensics, J. Anal. At. Spectrom. 26 (2011) 536-541, doi: 10.1039/C0JA00199F.

[9] D. A. Cremers, A. Beddingfield, R. Smithwick, R. C. Chinni, C. R. Jones, B. Beardsley, L. Karch, Monitoring Uranium, Hydrogen, and Lithium and Their Isotopes Using a Compact Laser-Induced Breakdown Spectroscopy (LIBS) Probe and High-Resolution Spectrometer, Appl. Spectrosc. 66 (2012) 250-261, doi: 10.1366/11-06314.

[10] X. Mao, G. C.-Y. Chan, I. Choi, V. Zorba, R. E. Russo, Combination of atomic lines and molecular bands for uranium optical isotopic analysis in laser induced plasma spectrometry, $\mathrm{J}$. Radioanal. Nucl. Chem. 312 (2017) 121-131, doi: 10.1007/s10967-017-5197-y.

[11] R. R. Hark, R. S. Harmon, S. Musazzi, U. Perini, Geochemical Fingerprinting Using LIBS, Eds. Berlin, Heidelberg: Springer Berlin Heidelberg, 2014, 309-348.

[12] R. S. Harmon, J. Remus, N. J. McMillan, C. McManus, L. Collins, J. L. Gottfried Jr., F. C. Delucia, A. W. Miziolek, LIBS analysis of geomaterials: Geochemical fingerprinting for the rapid analysis and discrimination of minerals, Appl. Geochem. 24 (2009) 1125-1141, doi: 10.1016/j.apgeochem.2009.02.009.

[13] A. I. Whitehouse, J. Young, I. M. Botheroyd, S. Lawson, C. P. Evans, J. Wright, Remote material analysis of nuclear power station steam generator tubes by laser-induced breakdown spectroscopy, Spectrochim. Acta B 56 (2001) 821-830, doi: 10.1016/S0584-8547(01)002324.

[14] C. Lee, On-site Laser Induced Breadown Spectroscopy (LIBS) for post-detonation nuclear forensics in real time, Thesis, Graduate school of UNIST, Ulsan, 2018. Accessed on: Nov. 29, 2019 [Online]. Available: https://scholarworks.unist.ac.kr/handle/201301/23559 
[15] C. Hernandez, H. Roche, C. Pocheau, C. Grisolia, L. Gargiulo, A. Semerok, Development of a Laser Ablation System Kit (LASK) for Tokamak in vessel tritium and dust inventory control, Fusion Eng. Des. 84 (2009) 939-942, doi: 10.1016/j.fusengdes.2008.12.033.

[16] W. Pietsch, A. Petit, A. Briand, Isotope ratio determination of uranium by optical emission spectroscopy on a laser-produced plasma - basic investigations and analytical results, Spectrochim. Acta B At. Spectrosc. 53 (1998) 751-761, doi: 10.1016/S0584-8547(97)001237.

[17] C. A. Smith, M. A. Martinez, D. K. Veirs, D. A. Cremers, Pu-239/Pu-240 isotope ratios determined using high resolution emission spectroscopy in a laser-induced plasma, Spectrochim. Acta B 57 (2002) 929-937, doi: 10.1016/S0584-8547(02)00023-X.

[18] M. Miyabe, M. Oba, K. Jung, H. limura, K. Akaoka, M. Kato, H. Otobe, A. Khumaeni, I. Wakaida, Laser ablation absorption spectroscopy for isotopic analysis of plutonium: Spectroscopic properties and analytical performance, Spectrochim. Acta B 134 (2017) 42-51, doi: 10.1016/j.sab.2017.05.008.

[19] B. W. Smith, I. B. Gornushkin, L. A. King, J. D. Winefordner, A laser ablation-atomic fluorescence technique for isotopically selective determination of lithium in solids, Spectrochim. Acta B 53 (1998) 1131-1138, doi: 10.1016/S0584-8547(98)00132-3.

[20] H. Niki, T. Yasuda, I. Kitazima, Measurement Technique of Boron Isotopic Ratio by Laserinduced Breakdown Spectroscopy, J. Nucl. Sci. Technol. 35 (1998) 34-39, doi: 10.3327/jnst.35.34.

[21] R. E. Russo, A. A. Bol'shakov, X. Mao, C. P. McKay, D. L. Perry, O. Sorkhabi, Laser Ablation Molecular Isotopic Spectrometry, Spectrochim. Acta B 66 (2011) 99-104, doi: 10.1016/j.sab.2011.01.007.

[22] A. Sarkar, X. Mao, R. E. Russo, Advancing the analytical capabilities of laser ablation molecular isotopic spectrometry for boron isotopic analysis, Spectrochim. Acta B 92 (2014) 42-50, doi: 10.1016/j.sab.2013.12.001.

[23] R. D. Cowan and G. H. Dieke, Self-Absorption of Spectrum Lines, Rev. Mod. Phys., 20 (1948), 418-455, doi: 10.1103/RevModPhys.20.418.

[24] A. Rastegari, M. Lenzner, C. Feng, L. Arissian, J. C. Diels, K. Peterson, Exploiting shock wave and self-absorption for high resolution laser induced breakdown spectroscopy, in 2017 Conference on Lasers and Electro-Optics (CLEO), San Jose, May 2017, pp. 1-2.

[25] C. Aragón, J. A. Aguilera, Determination of the local electron number density in laserinduced plasmas by Stark-broadened profiles of spectral lines: Comparative results from $\mathrm{Ha}$, Fe I and Si II lines, Spectrochim. Acta B 65 (2010) 395-400.

[26] J. S. Cowpe, R. D. Pilkington, J. S. Astin, A. E. Hill, The effect of ambient pressure on laser-induced silicon plasma temperature, density and morphology, J. Phys. Appl. Phys. 42 (2009) 165202, doi: 10.1088/0022-3727/42/16/165202.

[27] J. Bengoechea, J. A. Aguilera, C. Aragón, Application of laser-induced plasma spectroscopy to the measurement of Stark broadening parameters, Spectrochim. Acta B 61 (2006) 69-80, doi: 10.1016/j.sab.2005.11.003.

[28] M. Burger, M. Skočić, Z. Nikolić, S. Bukvić, S. Djeniže, Broadening of the resonance Cu I lines in the laser-induced copper spectrum, J. Quant. Spectrosc. Radiat. Transf. 133 (2014) 589-595, doi: 10.1016/j.jqsrt.2013.09.022. 
[29] A. D. Giacomo, J. Hermann, Laser-induced plasma emission: from atomic to molecular spectra, J. Phys. Appl. Phys. 50 (2017) 183002, doi: 10.1088/1361-6463/aa6585.

[30] J. Hermann, A. Lorusso, A. Perrone, F. Strafella, C. Dutouquet, B. Torralba, Simulation of emission spectra from nonuniform reactive laser-induced plasmas, Phys. Rev. E 92 (2015) 53103, doi: 10.1103/PhysRevE.92.053103.

[31] R. H. Hughes, Isotope Shift in the First Spectrum of Atomic Lithium, Phys. Rev. 99 (1955) 1837-1839, doi: 10.1103/PhysRev.99.1837.

[32] J. Walls, R. Ashby, J. J. Clarke, B. Lu, W. A. van Wijngaarden, Measurement of isotope shifts, fine and hyperfine structure splittings of the lithium D lines, Eur. Phys. J. - At. Mol. Opt. Plasma Phys. 22 (2003) 159-162, doi: 10.1140/epjd/e2003-00001-5.

[33] C. J. Sansonetti, B. Richou, R. Engleman, L. J. Radziemski, Measurements of the resonance lines of $6 \mathrm{Li}$ and $7 \mathrm{Li}$ by Doppler-free frequency-modulation spectroscopy, Phys. Rev. A 52 (1995) 2682-2688, doi: 10.1103/PhysRevA.52.2682.

[34] G. L. Stukenbroeker, D. D. Smith, G. K. Werner, J. R. McNally, Spectro-Isotopic Assay of Lithium*, JOSA 42 (1952) 383-386, doi: 10.1364/JOSA.42.000383.

[35] R. Noll, Laser-Induced Breakdown Spectroscopy, Ed. Springer Berlin Heidelberg, 2012, 7-15.

[36] M. Boueri, M. Baudelet, J. Yu, X. Mao, S. S. Mao, R. Russo, Early stage expansion and time-resolved spectral emission of laser-induced plasma from polymer, Appl. Surf. Sci. 255 (2009) 9566-9571, doi: 10.1016/j.apsusc.2009.04.088.

[37] J. Hermann, Ideal radiation source for plasma spectroscopy generated by laser ablation, Phys. Rev. E 96 (2017) 053210, doi: 10.1103/PhysRevE.96.053210.

[38] J. Puric, J. Labat, L. Cirkovic, I. Lakicevic, S. Djenize, Stark broadening and shift of alkalimetal resonance spectral lines, J. Phys. B At. Mol. Phys. 10 (1977) 2375, doi: 10.1088/0022$3700 / 10 / 12 / 016$.

[39] A. Bogaerts, Z. Chen, R. Gijbels, A. Vertes, Laser ablation for analytical sampling: what can we learn from modeling?, Spectrochim. Acta B 58 (2003) 1867-1893, doi: 10.1016/j.sab.2003.08.004. 